gonococcal porA pseudogene and multi-copy opa genes. Cycle threshold $(\mathrm{Ct})$ values obtained were used as semi-quantitative measures of gonococcal DNA. Sampling adequacy was assessed using a real-time PCR for human endogenous retrovirus 3 (ERV3).

Results 100 MSM with culture positive pharyngeal gonorrhoea were included. Isolation rates by culture from the tonsils and posterior oropharynx were $62 \%$ and $52 \%$ respectively $(\mathrm{p}=$ 0.041). PCR was significantly more sensitive than culture at both the tonsils $(84 \%$ vs. $62 \%$; $<0.001)$ and oropharynx $(81 \%$ vs. $52 \% ; \mathrm{p}<0.001)$. Culture positivity was greater with higher gonococcal DNA loads at both the tonsils $(\mathrm{p}=0.001)$ and oropharynx ( $\mathrm{p}<0.001)$. At the oropharynx, higher ERV3 DNA load was associated with improved gonococcal detection using culture $(p=0.013)$ as well as PCR $(p=0.045)$. At the tonsils, higher ERV3 DNA load was associated with improved gonococcal detection by PCR $(\mathrm{p}=0.040)$.

Conclusion Neisseria gonorrhoeae can be cultured from the tonsils as well as the posterior oropharynx with greater isolation rates where gonococcal loads are higher. While PCR is substantially more sensitive than culture at each site, like culture, PCR is dependent on the adequacy of sampling.

Disclosure of interest statement None to disclose.

\section{P07.13 DETECTION OF TREPONEMA PALLIDUM DNA FROM WHOLE BLOOD AND EARLOBE SPECIMENS IN PATIENTS FROM TWO STI CLINICS IN LIMA, PERU}

${ }^{1} \mathrm{JY} \mathrm{Chow}^{*},{ }^{2} \mathrm{JA}$ Flores, ${ }^{2} \mathrm{SK}$ Vargas, ${ }^{2} \mathrm{SR}$ Leon, ${ }^{1,2} \mathrm{KA}$ Konda, ${ }^{1} \mathrm{JD}$ Klausner, ${ }^{2} \mathrm{CF}$ Caceres. ${ }^{1}$ Division of Infectious Diseases, Division of Infectious Diseases, School of Medicine, University of California, Los Angeles, CA, USA; ${ }^{2}$ Unit of Health, Sexuality and Human Development, and Laboratory of Sexual Health, Universidad Peruana Cayetano Heredia, Lima, Peru

\subsection{6/sextrans-2015-052270.329}

Background Prior studies have reported PCR detection of Treponema pallidum DNA from whole blood and earlobe scrapings. Factors affecting efficiency such as PCR target gene and clinical stage of syphilis have been studied. We aimed to determine other factors associated with detection of T.pallidum DNA from whole blood and earlobe samples.

Methods Data were obtained from baseline samples collected for a prospective cohort study of 401 men who have sex with men (MSM) and transgender women (TW) in Lima, Peru. Participants were assessed for HIV using rapid HIV testing (Alere Determine, USA), a combined Antigen/Antibody HIV EIA, and Western blot confirmation (Genscreen ULTRA HIV Ag-Ab and Genetic Systems HIV-1 Western Blot, Bio Rad, USA). Syphilis infection was assessed with RPR (BD Macro-Vue, USA) and TPPA (Fujirebio, Japan). Whole blood samples and earlobe capillary blood was collected from patients with a positive RPR and TPPA. DNA extraction was performed using the QIAamp mini kit (Qiagen, Valencia, CA), and samples were then concentrated. Ujchowsing specific primers for the TpN47 gene, an aliquot of the DNA sample was amplified using conventional PCR for participants with high RPR titers $(\geq 1: 16)$. Positivity was determined by visual detection of PCR product on 1\% agarose gel.

Results 56 participants had RPR titer $\geq 1: 16(1: 16(\mathrm{n}=18), 1: 32$ $(\mathrm{n}=16), 1: 64(\mathrm{n}=17), 1: 128(\mathrm{n}=3)$, and $1: 256(\mathrm{n}=2))$. A total of $7(12.5 \%)$ participants had T.pallidum DNA detected from whole blood, and 6 (10.7\%) from earlobe capillary blood
(10 participants total). Of these 10 participants, 5 were HIV positive, and 5 were HIV negative.

Conclusion Our relatively low efficiency of detection from both whole blood and earlobe samples suggest that neither HIV status nor high RPR titer $(\geq 1: 16)$ seem to predict high likelihood of DNA detection. Further study is ongoing to determine if these or other characteristics are associated with positive detection of T.pallidum DNA.

Disclosure of interest statement The Picasso study is funded by a grant from the United States NIAID and was implemented by the Universidad Peruana Cayetano Heredia in collaboration with the University of California, Los Angeles. The molecular part of the study was implemented under the supervision of the University of Washington. No pharmaceutical grants were received during the development of this study.

\section{P07.14 APTIMA TV NAAT TEST PERFORMANCE IN GUM CLINICS AND PRIMARY CARE IN THE UK}

1] Nicholls ${ }^{*},{ }^{2} \mathrm{P}$ Muir, ${ }^{2} \mathrm{P}$ North, ${ }^{2} \mathrm{R}$ Ferguson, ${ }^{3} \mathrm{M}$ May, ${ }^{3} \mathrm{~K}$ Turner, ${ }^{3} \mathrm{~J}$ Macleod, ${ }^{1,3} \mathrm{P}$ Horner. ${ }^{1}$ Bristol Sexual Health Centre, University Hospitals Bristol NHS Trust, Bristol, UK; ${ }^{2}$ Public Health Laboratory Bristol, Public Health England, Bristol, UK; ${ }^{3}$ School of Social and Community Medicine, University of Bristol, Bristol, UK

\subsection{6/sextrans-2015-052270.330}

Introduction The Aptima TV NAAT for the detection of Trichomonas vaginalis (TV) is $\sim 100 \%$ sensitive compared to wet mount microscopy (50\%) or culture (75\%). Asymptomatic women attending genitourinary medicine (GUM) clinics and patients in primary care are often not tested for TV, as the prevalence and sensitivity of current tests is assumed to be too low for testing to be cost effective.

Our aim was to determine how many additional cases were identified with the new test and whether self-taken and cliniciantaken vaginal swabs are of equivalent sensitivity in symptomatic GUM patients.

Methods Patients were tested using the Aptima TV NAAT alongside existing testing methods.

Results The positivity of TV determined by TV NAAT was $4.8 \%$ $(26 / 543)$ and $1.8 \%(28 / 1593)$ in women with and without symptoms attending GUM and 2.7\% (95/3512) and 1.1\% (41/3593) respectively in primary care.

TV NAAT significantly outperformed our existing testing methods. In a subset of 491 GUM patients in whom all tests were performed, 14/23 (61\%) TV NAAT positive patients were identified on wet prep or culture $(p=0.004)$. In a subset of 2930 primary care patients in whom all tests were performed, 19/74 (26\%) TV NAAT positive patients were identified on wet prep or culture $(\mathrm{p}<0.0001)$. These results may reflect deterioration of specimens in transport to the laboratory.

Self- taken vaginal swabs were equivalent in sensitivity to clinician taken swabs; of the 26 patients who tested positive on either NAAT test, 25 tested positive on self-taken swab and 21 tested positive on clinician taken swab $(\mathrm{p}=0.51)$.

Conclusion Testing women attending GUM clinics and in primary care with the Aptima TV NAAT test will identify additional cases and should replace conventional microbiological testing methods if found to be cost-effective.

Disclosure of interest statement Hologic provided the tests for the Aptima TV NAAT research study and have sponsored the authors to present this data at ISSTDR. 